\title{
Consumption of olive oil has opposite effects on plasma total cholesterol and sphingomyelin concentrations in rats
}

\author{
Math J. H. Geelen ${ }^{1}$ and Anton C. Beynen ${ }^{2}$ \\ ${ }^{1}$ Laboratory of Veterinary Biochemistry and ${ }^{2}$ Departments of Nutrition and Laboratory Animal Science, \\ Graduate School of Animal Health, Faculty of Veterinary Medicine, Utrecht University, Utrecht, The Netherlands
}

(Received 19 November 1998 - Revised 13 September 1999 - Accepted 26 October 1999)

\begin{abstract}
The hypothesis that olive-oil consumption alters plasma sphingomyelin concentrations and hepatic sphingomyelin metabolism was tested. Rats were fed on purified, high-cholesterol diets with either coconut fat or olive-oil $(180 \mathrm{~g} / \mathrm{kg})$. In accordance with previous work, olive-oil $v$. coconut-fat consumption significantly elevated hepatic and total plasma cholesterol concentrations. During the course of the experiment, the concentration of plasma sphingomyelin rose in the coconut-fat group and remained constant in the olive-oil group. When compared with the coconut-fat-fed group, the plasma sphingomyelin levels were significantly lower in the olive-oilfed group after 14 and $21 \mathrm{~d}$ of treatment. Dietary olive oil raised the amounts of cholesterol and sphingomyelin in the VLDL density region, and this change was associated with a reduction in the cholesterol and sphingomyelin contents of the LDL and HDL density ranges. Olive-oil consumption reduced the activity of serine palmitoyltransferase, while the activities of phosphatidylcholine:ceramide cholinephosphotransferase and phosphatidylethanolamine:ceramide ethanolaminephosphotransferase were left unchanged. Dietary olive oil also enhanced the activity of acidic sphingomyelinase, but not that of neutral sphingomyelinase. The present data indicate that dietary olive oil $v$. coconut fat has opposite effects on total plasma cholesterol and sphingomyelin concentrations. The lower plasma sphingomyelin levels observed in olive-oil-fed, as compared with coconut-fat-fed rats, may be explained by a simultaneous elevation and reduction in sphingomyelin catabolism and synthesis respectively, as based on the measured enzyme activities.
\end{abstract}

\section{Sphingomyelin: Olive oil: Lipoproteins}

Sphingomyelin is a component of plasma lipoproteins (Merrill et al. 1995). Previous work has identified a positive correlation between the concentrations of sphingomyelin and cholesterol in whole plasma (Noël et al. 1972; Rodrigues et al. 1976; Merrill \& Jones, 1990). In a recent study with rats fed on a cholesterol-rich diet we corroborated the correlation and showed that the cholesterol-induced elevation in plasma sphingomyelin concentration was associated with a reduction in hepatic sphingomyelin catabolism (Geelen et al. 1995). We have hypothesized that cholesterol feeding causes an increase in hepatic sphingomyelin secretion which is secondary to an increase in VLDL production, because sphingomyelin is an essential structural component of VLDL (Geelen et al. 1995). This hypothesis could imply that conditions leading to a decrease in the availability of hepatic sphingomyelin would be associated with a decrease in VLDL secretion. If this reasoning is correct, plasma VLDL concentrations and possibly also cholesterol concentrations may be controlled through influencing sphingomyelin metabolism. Thus, further knowledge of the metabolic link between cholesterol and sphingomyelin metabolism is of both fundamental and applied interest.

The feeding of diets containing olive oil to rats causes an increase in the concentration of plasma cholesterol (Beynen, 1989). It could be suggested that olive-oil feeding has effects on sphingomyelin metabolism similar to those of a cholesterol challenge. To test this suggestion, we fed rats on diets containing either coconut fat or olive oil and determined sphingomyelin levels in plasma, liver and plasma lipoprotein fractions and also measured the activities of hepatic enzymes involved in the synthesis or catabolism of sphingomyelin.

\section{Materials and methods}

The experimental protocol was approved by the animal experiments committee of the Utrecht Faculty of Veterinary Medicine. 


\section{Chemicals}

$\mathrm{C}_{6}$-NBD ceramide was purchased from Molecular Probes (Eugene, OR, USA). The origin of other chemicals has been described previously (Geelen et al. 1995).

\section{Animals and diets}

Female outbred Wistar rats (HsdCpb:Wu, Harlan-CPB, Zeist, The Netherlands), aged 3 weeks, were used. They were housed in groups of three per cage in an animal room with a $12 \mathrm{~h}$ light-dark cycle (lights on, 07.00-19.00 hours). All rats were fed on a diet containing coconut fat for $7 \mathrm{~d}$. The composition of the diet was as follows $(\mathrm{g} / \mathrm{kg})$ : coconut fat, 180; maize oil, 20; cholesterol, 10; casein, 200; sucrose, 200; maize starch, 301; cellulose, 30; $\mathrm{CaCO}_{3}, 12$; monosodium phosphate, $15 ; \mathrm{MgCO}_{3}, 2 ; \mathrm{KCl}, 8$; mineral premix, 10 ; vitamin premix 12 . The compositions of the vitamin and mineral premixes have been described by Verbeek et al. (1993). After $7 \mathrm{~d}$ (day 0 of the experiment) the rats were divided into two groups of eighteen rats each and one group of six rats, which were stratified for body weight. One group of eighteen rats continued to receive the diet with coconut fat and the other group of eighteen rats was transferred to the same diet containing olive oil $(180 \mathrm{~g} / \mathrm{kg})$ instead of coconut fat. The diets were formulated to elicit a marked hypercholesterolaemic response to olive oil (Beynen, 1987). Animals had free access to food, which was in powdered form, and tap water.

\section{Collection and preparation of samples}

Blood and liver samples were taken exactly as described before (Geelen et al. 1995). Lipoproteins were isolated from fresh plasma by density gradient centrifugation (Terpstra et al. 1981). On the basis of their density $(d, \mathrm{~kg} / \mathrm{l})$, VLDL $(d<1.006)$, LDL $(1.019<d<1.063)$ and HDL-2 $(1.063<$ $d<1.125)$ were collected. Isolated lipoprotein fractions were frozen and stored at $-20^{\circ}$ until analysis.

Several pieces of liver were homogenized separately and used for lipid extraction and subcellular fractionation as described previously (Geelen et al. 1995).

\section{Enzyme assays}

Activities of sphingomyelinase were determined in liver preparations by measuring the release of phospho[methyl- ${ }^{14} \mathrm{C}$ ]choline from [choline-methyl- ${ }^{14} \mathrm{C}$ ]sphingomyelin. Acidic sphingomyelinase activity was assayed in liver homogenates as described before (Geelen et al. 1995). Assays were conducted at $\mathrm{pH} 4.4$ for $60 \mathrm{~min}$ at $37^{\circ}$. Neutral sphingomyelinase activity was determined in isolated plasma membranes. The latter assay was performed as the one for acidic sphingomyelinase except that the buffer was $50 \mathrm{mM}$-Tris- $\mathrm{HCl}(\mathrm{pH} \mathrm{7.4)}$ and the incubation was carried out in the presence of $40 \mathrm{mM}-\mathrm{MgCl}_{2}$ for $20 \mathrm{~min}$.

Determination of the activity of serine palmitoyltransferase was based on the incorporation of $\left[{ }^{3} \mathrm{H}\right]$ serine into chloroform-soluble products as described by Williams et al. (1984).

The activities of phosphatidylcholine:ceramide cholinephosphotransferase and phosphatidylethanolamine:ceramide ethanolaminephosphotransferase were determined essentially as described by Vos et al. (1995). Briefly, the assay mixture contained in a total volume of $250 \mu \mathrm{l}: 26 \mu \mathrm{mol} / 1 \mathrm{C}_{6}$-NBD ceramide, $174 \mu \mathrm{mol} / \mathrm{l}$ egg phosphatidylcholine or phosphatidylethanolamine, $50 \mathrm{mmol} / \mathrm{l}$ Tris- $\mathrm{HCl}$ ( $\mathrm{pH} 7 \cdot 4$ ), $5 \mathrm{mmol} / \mathrm{l}$ EDTA, $20 \mu \mathrm{g}$ Triton X-100 and $50 \mu \mathrm{l}$ purified plasma membrane. Control experiments demonstrated that the assays were linear with protein up to at least $200 \mu \mathrm{g}$ plasma membrane protein and with time for at least $3 \mathrm{~h}$. For routine purposes, assays were conducted for $60 \mathrm{~min}$ with about $100 \mu \mathrm{g}$ plasma membrane protein. Lipids were extracted according to the method of Bligh \& Dyer (1959) and separated by TLC on silica G-60 plates using chloroformmethanol-250 g/l $\mathrm{NH}_{4} \mathrm{OH}-$ water (70:30:4:1, by vol.) as developing solvent. NBD-sphingomyelin spots were detected under u.v.-light and scraped from the plates. For quantification, NBD-sphingomyelin was excited at $465 \mathrm{~nm}$ and its fluorescence was measured at $530 \mathrm{~nm}$. The fluorimetry was carried out with a Perkin Elmer Luminescence Spectrometer LS-50 (Perkin Elmer, Beaconsfield, Bucks., UK).

\section{Chemical analyses}

Cholesterol and sphingomyelin in plasma, liver homogenates and lipoprotein fractions were isolated and quantified exactly as described previously (Geelen et al. 1995).

\section{Statistical analysis}

Results shown represent means and standard deviations. The data within the two dietary groups for the three time points were independent so that statistical analysis of diet effects for each time point was performed by two-tailed Student's $t$ test. The combined data for days 7, 14 and 21 were also subjected to factorial ANOVA to disclose any diet effects. The level of significance was pre-set at $P<0 \cdot 05$.

\section{Results}

Body weights of the control and olive-oil-fed rats did not differ significantly; on day 21 of the experiment the values were 156.4 (SD 8.4) and 165.8 (SD 8.4) g respectively $(n 6)$. Olive oil did not significantly affect liver weight. On day 21 of the experiment, relative liver weights in the control and olive-oil-fed groups were 50.1 (SD 2.09) and 52.0 (SD 1.04) $\mathrm{g} / \mathrm{kg}$ body weight respectively.

Replacement of coconut fat by olive oil caused elevated concentrations of total cholesterol in plasma and liver (Fig. 1). In the course of the experiment, plasma sphingomyelin concentrations rose in the coconut-fat group, but remained constant in the olive-oil-fed group. Consequently, olive-oil $v$. coconut-fat feeding significantly reduced sphingomyelin concentrations. The amount of sphingomyelin in liver decreased during the course of the experiment, but there was no effect of the type of dietary fat (Fig. 1(d)).

As shown in Fig. 2, olive-oil feeding significantly elevated the amount of cholesterol in the VLDL fraction, but lowered LDL- and HDL-cholesterol concentrations. Consumption of the diet containing olive oil induced a rapid rise in the sphingomyelin concentration of VLDL (Fig. 2(b)) and reduced LDL-sphingomyelin by $50 \%$ after 14 and $21 \mathrm{~d}$ (Fig. 2(d)). Olive oil $v$. coconut fat induced a marked drop 

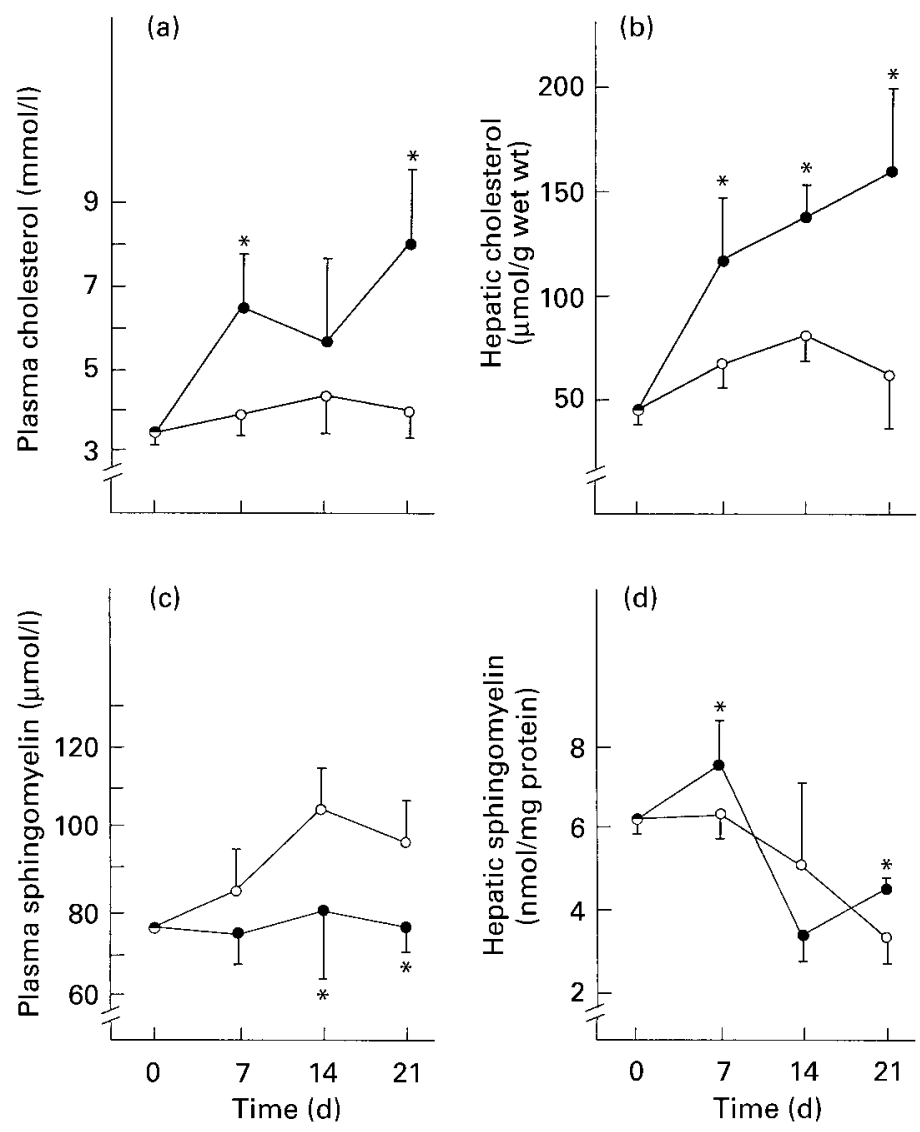

Fig. 1. (a) Plasma cholesterol, (b) hepatic cholesterol, (c) plasma sphingomyelin and (d) hepatic sphingomyelin concentrations in rats fed on a diet containing either coconut fat $(\mathrm{O})$ or olive oil $(\bullet)$ for $21 \mathrm{~d}$. Values are means for six rats, with standard deviations represented by vertical bars. Mean values were significantly different from those for the coconut-fat diet: ${ }^{\star} P<0.05$. For all data combined, ANOVA disclosed significant $(P<0.05)$ diet effects for plasma cholesterol and sphingomyelin and for liver cholesterol.

in cholesterol concentrations in HDL (Fig. 2(e)). HDLsphingomyelin concentrations were reduced by olive-oil feeding after 7 and $21 \mathrm{~d}$ (Fig. 2(f)).

We determined the hepatic activities of a number of key enzymes of the synthesis and degradation of sphingomyelin. The results in Fig. 3 show that consumption of olive oil lowered the activity of serine palmitoyltransferase. As shown in Fig. 4, olive-oil feeding did not systematically affect the activities of the sphingomyelin synthesizing enzymes in hepatic plasma membranes, phosphatidylcholine:ceramide phosphocholinetransferase and phosphatidylethanolamine: ceramide phosphoethanolaminetransferase. The activities of the acidic and neutral forms of sphingomyelinase were higher at two time points after olive-oil feeding, but the difference only reached significance for acidic sphingomyelinase at day 21 (Fig. 5). ANOVA showed that olive oil $v$. coconut fat significantly elevated acidic sphingomyelinase activity.

\section{Discussion}

In the present study with rats, we examined the effect of olive oil $v$. coconut fat consumption on the plasma concentrations and hepatic metabolism of sphingomyelin. In accordance with previous observations (Beynen, 1987,
1989) the animals fed with olive oil accumulated substantially more cholesterol in their livers and blood plasma than did the animals fed with coconut fat. The novel and unexpected outcome of this study is that olive-oil feeding reduced the amount of sphingomyelin in plasma. Thus, the earlier reported positive correlations between plasma total cholesterol and sphingomyelin (Noël et al. 1972; Rodrigues et al. 1976; Merrill and Jones 1990) did not extend to rats fed on a diet containing olive oil. Hepatic sphingomyelin concentrations also showed a pattern that differed from that of cholesterol. Olive oil $v$. coconut fat did not affect hepatic sphingomyelin concentrations. During the course of the experiment hepatic sphingomyelin stores were depleted, suggesting that sphingomyelin was mobilized. The changes in lipoprotein concentrations of cholesterol and sphingomyelin as induced by olive-oil feeding were positively correlated. After substitution of dietary olive oil for coconut fat, both cholesterol and sphingomyelin contents of VLDL rose whereas those of LDL and HDL fell. The rise in VLDLcholesterol and sphingomyelin may be explained by a depressed catabolism of VLDL particles (Cortese et al. 1983). Plasma transfer of sphingomyelin from LDL and HDL-2 to VLDL might also be responsible for the rise in VLDL sphingomyelin in the animals fed with olive oil. 


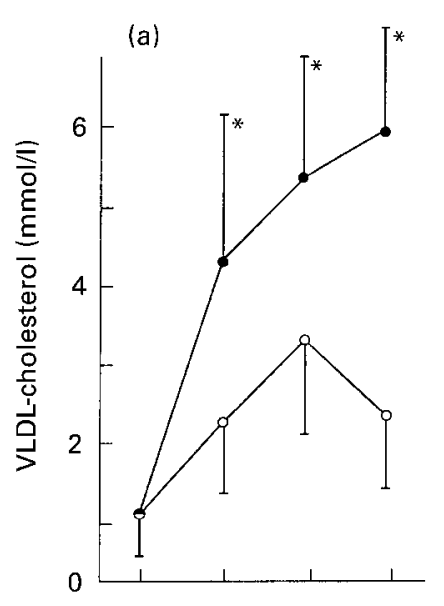

(b)
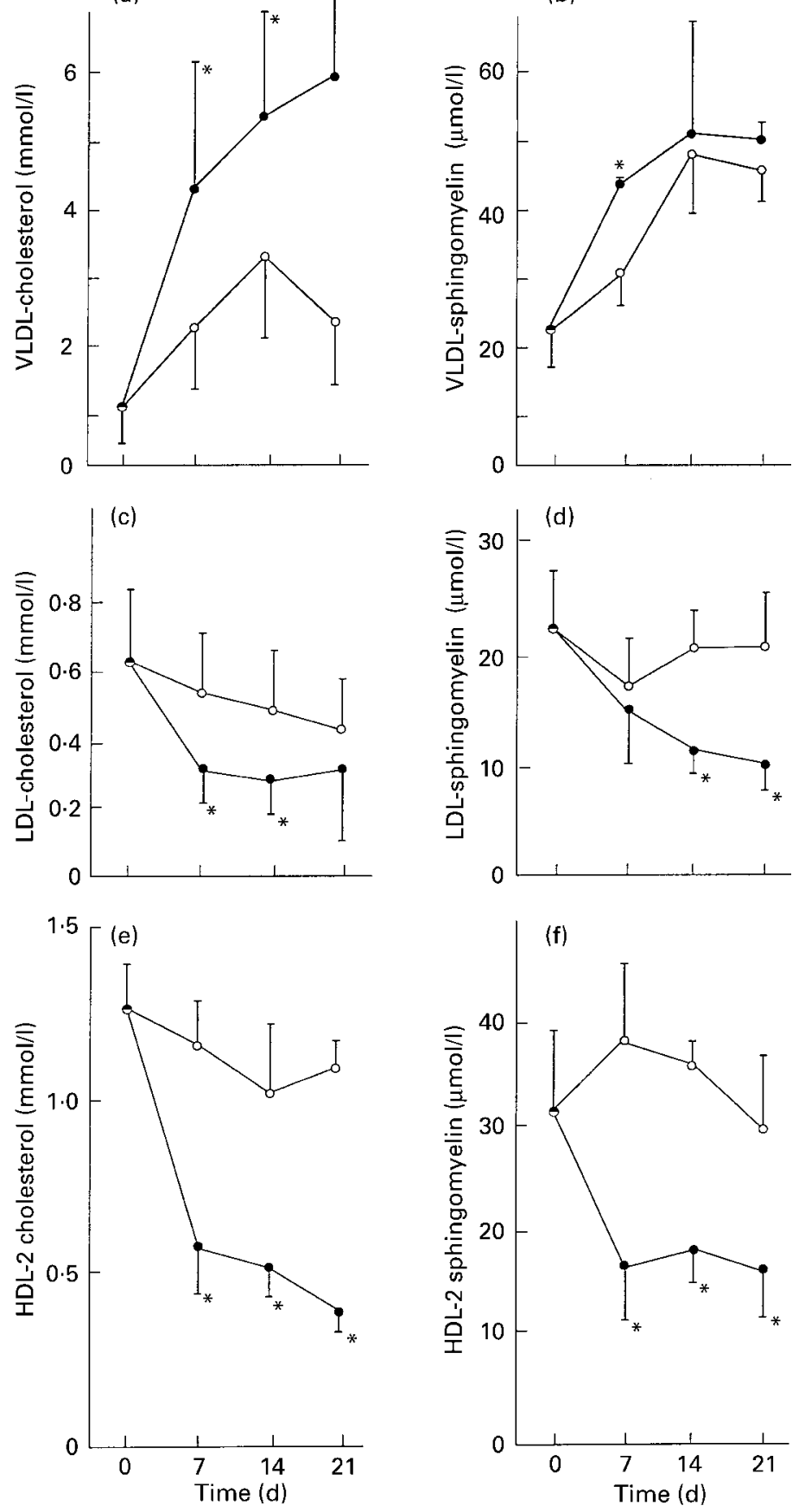

Fig. 2. (a, c, e) Cholesterol and (b, d, f) sphingomyelin concentrations in VLDL, LDL and HDL-2 fractions isolated from the plasma of rats fed on a diet containing either coconut fat $(O)$ or olive oil $(\bullet)$ for $21 \mathrm{~d}$. Samples from two rats were pooled; values are means of three pooled fractions, with standard deviations represented by vertical bars. Mean values were significantly different from those for the coconut-fat diet: ${ }^{*} P<0.05$. For all data combined, ANOVA showed significant $(P<0.05)$ diet effects for VLDLcholesterol, LDL-cholesterol, LDL-sphingomyelin and HDL-2 sphingomyelin.

However, in hamsters the feeding of olive oil instead of either palm oil or maize oil did not affect the activity of phospholipid transfer protein (Terpstra et al. 2000). The observed changes in LDL and HDL composition probably relate to the method of isolation of these lipoproteins which was based on a fixed density range. The olive-oil diet produced a fall in LDL-cholesterol, which can be explained because LDL is the product of intravascular VLDL catabolism, but seems aberrant as the feeding of olive oil promotes LDL clearance from the circulation by increasing hepatic receptor activity (Woollett et al. 1994). A lowering of LDLcholesterol has been observed before in studies with rats fed 


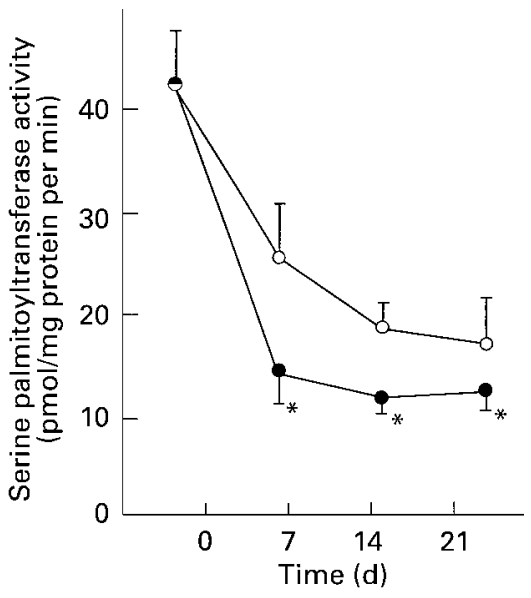

Fig. 3. Hepatic serine palmitoyltransferase activity in rats fed on diets containing either coconut fat $(O)$, or olive oil $(\bullet)$ for $21 \mathrm{~d}$. Values are means for six rats, with standard deviations indicated by vertical bars. Per liver sample, enzyme assays were carried out in triplicate. Mean values were significantly different from those for the coconut-fat diet: ${ }^{*} P<0.05$. For all data combined, ANOVA showed a significant $(P<0.05)$ effect of dietary fat type for serine palmitoyltransferase.

on a cholesterol-rich diet (Beynen et al. 1984; Geelen et al. 1995) and is most probably caused by a diet-induced decrease in the density of the LDL particles that are consequently recovered in the density range $<1.019 \mathrm{~kg} / \mathrm{l}$ (Beynen et al. 1984) and thus were not isolated with the LDL fraction in the present study. Likewise, some of the HDL particles may have shifted to the LDL density range, but this did not compensate for the LDL-cholesterol that had moved to the VLDL density range.

At the end of the experiment (day 21) we also measured, according to methods described earlier (Geelen et al. 1995), whole plasma concentrations of phospholipids other than sphingomyelin. For the rats fed with coconut fat and olive oil respectively, the concentrations of the major phospholipids were as follows $(n 6)$ : phosphatidylcholine, 1056 (SD 240) and 549 (SD 110) $\mu \mathrm{mol} / \mathrm{l}$ and lysophosphatidylcholine, 130 (SD 14.3) and 88.9 (SD 7.5) $\mu \mathrm{mol} / \mathrm{l}$. Olive-oil feeding significantly lowered total phospholipid concentrations $(P<0 \cdot 05)$. Thus, the decrease in sphingomyelin was associated with a decrease in total phospholipid concentration. However, it is difficult to conclude that the diet-induced changes reflect compensatory mechanisms to control the size of lipoprotein particles. In any event, it is clear that plasma sphingomyelin in the control group represents only about $7 \%$ of total plasma phospholipids.

The present data can provide clues as to whether the drop in whole plasma sphingomyelin seen after olive-oil feeding may be caused by changes in hepatic sphingomyelin synthesis and/or catabolism. Determination of the hepatic activity of serine palmitoyltransferase, key enzyme of sphingomyelin formation, indicated that dietary olive oil may decrease the biosynthesis of this phospholipid. In liver plasma membranes, the activities of the sphingomyelin-synthesizing enzymes phosphatidylcholine:ceramide phosphocholine transferase and phosphatidylethanolamine:ceramide phosphoethanolamine transferase were not affected by olive oil.
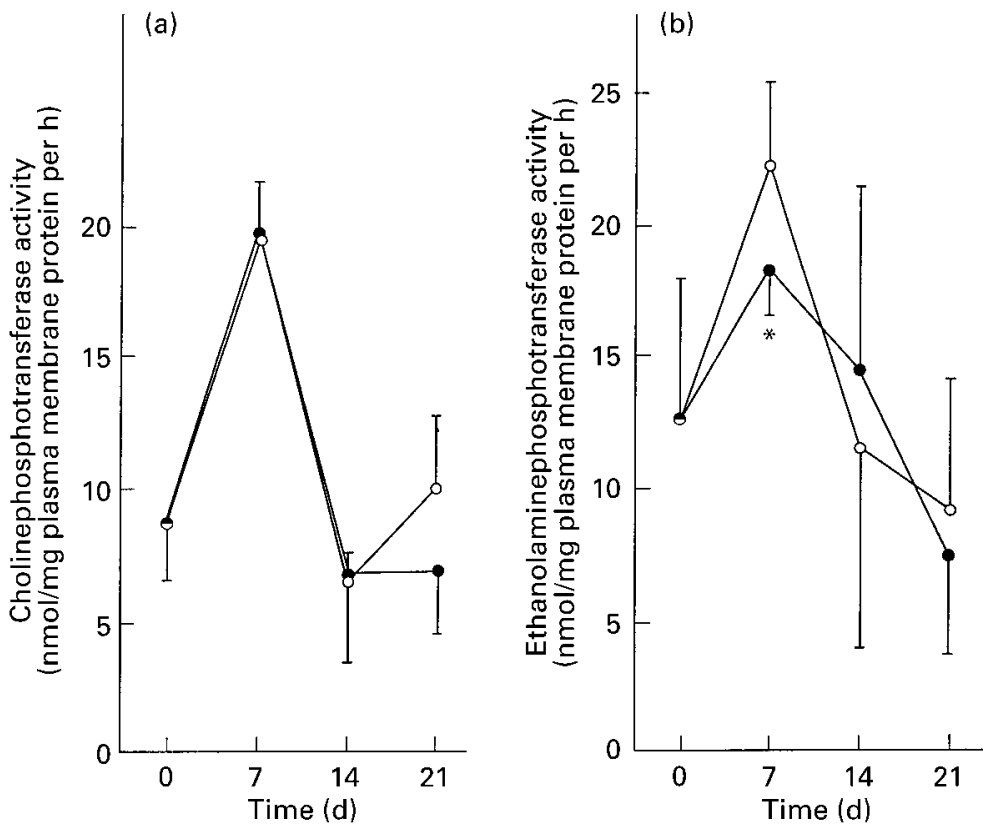

Fig. 4. Activities of (a) phosphatidylcholine:ceramide cholinephosphotransferase and (b) phosphatidylethanolamine:ceramide ethanolaminephosphotransferase in hepatic membranes of rats fed on a diet containing either coconut fat $(O)$, or olive oil $(\bullet)$ for $21 \mathrm{~d}$. Values are means for six rats, with standard deviations represented by vertical bars. Per liver sample, each enzyme assay was carried out in triplicate. Mean values were significantly different from those for the coconut-fat diet: ${ }^{\star} P<0.05$. For all data combined, ANOVA did not show significant effects of diet. 

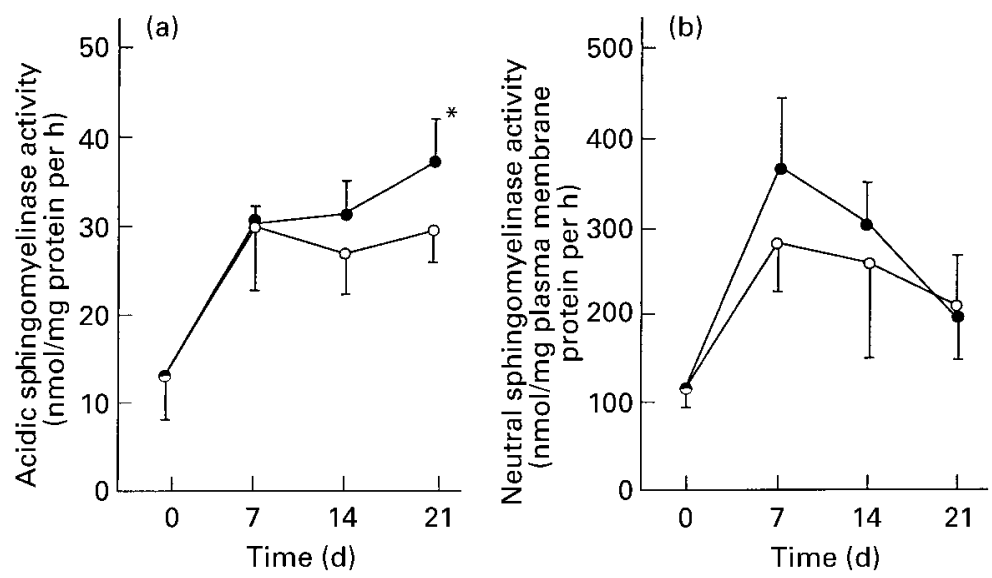

Fig. 5. Hepatic activities of sphingomyelinase in (a) lysosomes and (b) plasma membranes of rats fed on a diet containing either coconut fat $(O)$ or olive oil $(\bullet)$ for $21 \mathrm{~d}$. Values are means for six rats, with standard deviations represented by vertical bars. Per liver sample, each enzyme assay was carried out in triplicate. Mean values were significantly different from those for the coconut-fat diet: ${ }^{\star} P<$ 0.05 . For all data combined, ANOVA showed a significant $(P<0.05)$ diet effect for lysosomal sphingomyelinase.

On the other hand, the activity of the sphingomyelindegrading enzyme, acidic sphingomyelinase, was significantly raised by olive oil. From the changes in enzyme activities one would expect a drop in the hepatic and/or plasma level of sphingomyelin. Indeed, an olive-oil-induced lower concentration was observed in plasma, but not in VLDL or in liver.

In the two dietary groups there were decreases in hepatic sphingomyelin concentrations during the course of the feeding period. This change may be explained by the increase in the activity of acidic sphingomyelinase and a change of hepatic serine palmitoyltransferase activity which tended to decrease. It may be expected that a new steadystate concentration of hepatic sphingomyelin would be reached after continuous feeding of the diet containing olive oil.

In conclusion, the feeding of a diet containing olive oil instead of coconut fat produced a reduction in whole plasma sphingomyelin concentrations. This effect was probably caused by the consumption of oleic acid, the major fatty acid in olive oil, at the expense of lauric and myristic acids which occur abundantly in coconut fat (Beynen, 1987). The olive-oil-induced lowering of plasma sphingomyelin was associated with a rise in sphingomyelin in the VLDL density range and reduction in the LDL and HDL density ranges. The lowering of plasma total sphingomyelin after the feeding of olive oil may be explained by a lower activity of hepatic serine palmitoyltransferase and a higher hepatic activity of acidic sphingomyelinase.

\section{Acknowledgements}

We are most grateful to Danny van Hoorn and Inez Lemmens for excellent technical assistance. Supported in part by the Netherlands Foundation for Chemical Research (SON) with financial aid from the Netherlands Organization for Scientific Research (NWO).

\section{References}

Beynen AC (1987) Serum and liver cholesterol in rats fed cholesterol-free or high-cholesterol diets differing in type and amount of fat. Nutrition Reports International 35, 1327-1332.

Beynen AC (1989) Dietary olive oil and liver cholesterol in rats. Acta Alimentaria 18, 295-303.

Beynen AC, Boogaard A, Van Laack HLJM \& Katan MB (1984) Cholesterol metabolism in two strains of rats with high or low response of serum cholesterol to a standard cholesterol-rich diet. Journal of Nutrition 114, 1640-1651.

Bligh EG \& Dyer WJ (1959) A rapid method of total lipid extraction and purification. Canadian Journal of Biochemistry and Physiology 37, 911-917.

Cortese C, Levy Y, Janus ED, Turner PR, Rao SN, Miller NE \& Lewis B (1983) Modes of action of lipid-lowering diets in man: studies of apoliporotein B kinetics in relation to fat consumption and dietary fatty acid composition. European Journal of Clinical Investigation 13, 79-85.

Geelen MJH, Tijburg LBM, Bouma CJ \& Beynen AC (1995) Cholesterol consumption alters hepatic sphingomyelin metabolism in rats. Journal of Nutrition 125, 2294-2300.

Merrill AH Jr \& Jones DD (1990) An update of the enzymology and regulation of sphingomyelin metabolism. Biochimica et Biophysica Acta 1044, 1-12.

Merrill AH Jr, Lingrill S, Wang E, Nikolova-Karakashian M, Vales TR \& Vance DE (1995) Sphingolipid biosynthesis de novo by rat hepatocytes in culture. Ceramide and sphingomyelin are associated with, but not required for, very low density lipoprotein secretion. Journal of Biological Chemistry 270, 13834-13841.

Noël C, Marcel YL \& Davignon J (1972) Plasma phospholipids in the different types of hyperlipoproteinemia. Journal of Laboratory and Clinical Medicine 79, 611-621.

Rodrigues JL, Ghiselli GC, Torreggiani D \& Sirtori CR (1976) Very-low-density lipoproteins in normal and cholesterol-fed rabbits: lipid and lipoprotein composition and metabolism. Atherosclerosis 23, 73-83.

Terpstra AHM, Van den Berg P, Jansen H, Beynen AC \& Van Tol A (2000) Dietary palm, olive, and corn oil have different effects on plasma lipoprotein metabolism in hamsters. British Journal of Nutrition 83, 151-159. 
Terpstra AHM, Woodward CJH \& Sanchez-Muniz FJ (1981) Improved techniques for the separation of serum lipoproteins by density gradient ultracentrifugation: visualization by prestaining and rapid separation of serum lipoproteins from small volumes of serum. Analytical Biochemistry 111, 149-157.

Verbeek MJF, Van den Berg GJ, Lemmens AG \& Beynen AC (1993) High protein intake raises apparent but not true magnesium absorption in rats. Journal of Nutrition 123, 1880-1887.

Vos JP, Giudici ML, Van der Bijl P, Magni P, Marchesini S, Van Golde, LMG \& Lopes-Cardozo M (1995) Sphingomyelin is synthesized at the plasma membrane of oligodendrocytes and by purified myelin membranes: a study with fluorescentand radio-labelled ceramide analogues. FEBS Letters 368, 393396.

Williams RD, Wang E \& Merrill AH Jr (1984) Enzymology of long-chain base synthesis by liver: characterization of serine palmitoyltransferase in rat liver microsomes. Archives of Biochemistry and Biophysics 228, 282-291.

Woollett LA, Daumerie CM \& Dietschy JM (1994) Trans9-octadecenoic acid is biologically neutral and does not regulate the low density lipoprotein receptor as the cis isomer does in the hamster. Journal of Lipid Research 35, 1661-1673. 